\title{
CONTEXTO DA REDE DE RELAÇÕES E INTERAÇÕES DO AGENTE COMUNITÁRIO DE SAÚDE*
}

Gabriela Marcellino de Melo Lanzoni ${ }^{1}$, Aline Lima Pestana², Monique Haenscke Senna1, Betina Hörner Schlindwein Meirelles ${ }^{3}$

RESUMO: Este estudo objetivou compreender o contexto no qual se desenvolve a rede de relações e interações dos Agentes Comunitários de Saúde na Estratégia Saúde da Família. Utilizou-se a Teoria Fundamentada nos Dados como referencial metodológico; os dados foram coletados entre fevereiro e abril de 2009 em uma Unidade Local de Saúde de Florianópolis-Santa Catarina, por meio de entrevista com 21 participantes distribuídos em três grupos amostrais. Da análise destaca-se que a interação entre agentes comunitários, usuários e equipe acontece por meio da visita domiciliar, de reuniões, grupos terapêuticos e confraternização com a equipe. Conclui-se que o contexto das relações e interações dos agentes torna-se amplo, pois suas atividades são diretamente ligadas à comunidade, extrapolando os limites da Unidade Local. Aponta-se que os encontros informais promovidos pela equipe são importante estratégia para a formação de vínculo dentre profissionais e Agentes Comunitários de Saúde.

DESCRITORES: Enfermagem; Saúde da família; Atenção primária à saúde; Relações interpessoais; Relações interprofissionais.

\section{THE CONTEXT OF THE COMMUNITY HEALTH WORKER'S NETWORK OF RELATIONSHIPS AND INTER-RELATIONSHIPS}

\begin{abstract}
This study aimed to understand the context in which the Family Health Strategy Community Health Worker's network of relationships and inter-relationships develops. Grounded Theory was used as a methodological framework; the data was collected between February and April 2009 in a Local Health Unit in Florianópolis in Santa Catarina, through interviews held with 21 participants distributed in three sample groups. From the analysis, it stands out that the interaction between Community Health Workers (CHW), service users and the team takes place through home visits, meetings, therapeutic groups and socializing with the team. It is concluded that the CHWs' context of relationships and inter-relationships is broad, as their activities are directly linked to the community, overcoming the Local Unit's limitations. It is indicated that the informal meetings promoted by the team are an important strategy for forming the link between professionals and Community Health Workers.

DESCRIPTORS: Nursing; Family health; Primary health care; interpersonal relationships; inter-professional relationships.

\section{CONTEXTO DE LA RED DE RELACIONES E INTERACCIONES DEL AGENTE COMUNITARIO DE SALUD}

RESUMEN: Este estudio tuvo la finalidad de comprender el contexto en el cual se desarrolla la red de relaciones e interacciones de los Agentes Comunitarios de Salud en la Estrategia Salud de la Familia. Fue utilizada la Teoría Fundamentada en los datos como referencial metodológico; los datos fueron obtenidos entre febrero y abril de 2009 en una Unidad Local de Salud de Florianópolis, Santa Catarina, por medio de entrevista con 21 participantes organizados en tres grupos. Del análisis, se destaca que la interacción entre agentes comunitarios, usuarios y equipo ocurre por medio de la visita domiciliar, de reuniones, grupos terapéuticos y confraternización con el equipo. Se concluye que el contexto de las relaciones e interacciones de los agentes se vuelve amplio, pues sus actividades están directamente asociadas a la comunidad, extrapolando los límites de la Unidad Local. Se apunta que los encuentros informales promovidos por el equipo son importante estrategia para la formación de vínculo entre profesionales y Agentes Comunitarios de Salud. DESCRIPTORES: Enfermería; Salud de la Familia; Atención primaria a la salud; Relaciones interpersonales; Relaciones interprofesionales.

\footnotetext{
*Artigo elaborado a partir da dissertação de mestrado "Vislumbrando a rede complexa de relações e interações do Agente Comunitário de Saúde", apresentado ao PEN UFSC em 2009.

${ }^{1}$ Enfermeira. Mestre em Enfermagem. Doutoranda pelo Programa de Pós-Graduação em Enfermagem da Universidade Federal de Santa Catarina - PEN UFSC. Bolsista CAPES. Integrante do Grupo de Pesquisas em Administração e Gerência do Cuidado em Saúde e Enfermagem - GEPADES.

${ }^{2}$ Enfermeira. Mestre em Enfermagem. Doutoranda pelo PEN UFSC. Bolsista CNPq. Integrante do GEPADES.

${ }^{3}$ Enfermeira. Doutora em Enfermagem. Professora do Departamento e do PEN UFSC. Integrante do GEPADES. Bolsista de Produtividade em Pesquisa do CNPq.
}

Autor correspondente:

Gabriela Marcellino de Melo Lanzoni

Recebido: 01/10/2012

Universidade Federal de Santa Catarina

Rua José Ferreira Correa, 227 - 88090-040 - Florianópolis-SC-Brasil

E-mail: gabimrc@gmail.com 


\section{INTRODUÇÃO}

O Sistema Único de Saúde (SUS) foi instituído no Brasil na década de 1980 com o propósito de identificar e divulgar fatores condicionantes e determinantes à saúde, formular políticas destinadas a promover ações e serviços de saúde, bem como assistir as pessoas por meio de ações de promoção, proteção e recuperação da saúde. Nesta perspectiva, o SUS busca alternativas para melhorar a qualidade da assistência de acordo com as novas demandas. Com vistas à assistência à saúde da população, foi elaborada a Estratégia Saúde da Família (ESF), que tem como foco a qualificação e consolidação da Atenção Básica, ampliando a resolutividade frente às situações de saúde das pessoas e comunidades. Desta forma, almeja-se a integralidade da assistência ao usuário como sujeito inserido em um contexto familiar, vinculado ao domicílio e à comunidade, em uma atuação multiprofissional e interdisciplinar ${ }^{(1-3)}$.

A perspectiva da ESF faz com que a família passe a ser o objeto central de atenção, entendida a partir do ambiente onde vive. Este novo modelo de assistência prevê a utilização da visita domiciliar como forma de inserir o profissional na realidade do usuário e de sua família, como também proporcionar o estabelecimento de vínculos entre estes ${ }^{(2,4)}$.

A equipe de saúde é composta por um médico generalista, enfermeiro, auxiliar ou técnico em enfermagem e agente comunitário de saúde, sendo este a principal ferramenta para a consolidação dos objetivos propostos pela ESF, por trabalhar com o indivíduo e sua família, em uma área adstrita e ter acesso ao modo de vida desta população. Nesse contexto, acredita-se que o Agente Comunitário de Saúde (ACS) torna-se o articulador do processo de trabalho da equipe, exatamente por morar na sua área de atuação, conhecer a comunidade em que vive e ter maior facilidade de acesso aos domicílios ${ }^{(5)}$.

Por definição, o ACS trabalha fora da unidade, fazendo a ligação entre a comunidade e os serviços de saúde. $\mathrm{O}$ vínculo acontece de várias maneiras, mas é facilitado através da visita domiciliar, onde o ACS tem a oportunidade de conhecer os agravos que acometem a família, e então comunicar à equipe de saúde. Juntos, equipe de saúde e ACS realizarão intervenções necessárias para evitar, diminuir ou solucionar os problemas encontrados $^{(5)}$. A proximidade que é estabelecida por meio da visita domiciliar realizada pelo ACS favorece o levantamento das informações sobre a saúde individual e familiar daqueles com quem se relaciona na comunidade. Essa interação gera confiança entre os envolvi- dos, contribuindo para as decisões que serão planejadas e executadas no processo de trabalho em saúde ${ }^{(6-8)}$.

A prática interdisciplinar coloca-se como potencializadora da integração entre a equipe de saúde através da articulação entre os diversos saberes e fazeres presentes no trabalho em busca da saúde do usuário ${ }^{(9)}$. Assim, as equipes multiprofissionais são consideradas instrumento fundamental para o desenvolvimento de um trabalho em saúde de qualidade ${ }^{(10)}$.

Destaca-se a atuação do enfermeiro por ser um profissional muito influente na ESF, tanto no âmbito assistencial quanto administrativo. Este desenvolve atividades de supervisão e capacitação da equipe de enfermagem e dos ACS, como também realiza o gerenciamento de materiais e de pessoal na Unidade Básica de Saúde, sendo o eixo central do trabalho na equipe multiprofissional ${ }^{(11)}$.

Sabendo que a ESF consolida-se com a complementariedade do trabalho dos diferentes profissionais de saúde e dos ACS que nela atuam, para tanto, é necessário entender esta relação visando aprimorar o alcance das ações em saúde e sua qualidade. Desta forma, questiona-se: Como os ACS percebem e vivenciam o contexto da sua rede de relações e interações? Assim, o objetivo do estudo foi compreender o contexto no qual se desenvolve a rede de relações e interações dos ACS em uma Estratégia da Saúde da Família.

\section{MÉTODO}

Trata-se de uma pesquisa qualitativa, na qual foi adotado como referencial metodológico a Teoria Fundamentada nos Dados (TFD). Esta tem como objetivo desenvolver e relacionar conceitos, permitindo que uma teoria surja influenciada pelos dados descobertos, e não apenas pelas especulações do pesquisador, configurando um guia importante para a ação ${ }^{(12)}$.

Os dados foram coletados entre os meses de fevereiro a abril de 2009, em um centro de saúde de Florianópolis-Santa Catarina, por meio de entrevista semiestruturada, na própria instituição ou residência dos participantes. Utilizou-se como questão inicial: "Como você experiencia e confere significado às suas relações e interações?" O encaminhamento das demais questões foi direcionado pelas pesquisadoras, a partir das respostas dos entrevistados.

Participaram do estudo 21 pessoas, distribuídas em três grupos amostrais. O primeiro grupo amostral foi constituído por $8 \mathrm{ACS}$, o segundo grupo foi formado por dois médicos, dois enfermeiros, um dentista e qua- 
tro ACS. Já o terceiro grupo amostral foi constituído por quatro usuários do centro de saúde.

A análise se deu concomitantemente com a coleta dos dados. A codificação é o processo central do desenvolvimento do modelo teórico, ocorrendo em três etapas que acontecem simultaneamente: codificação aberta, axial, e seletiva. A codificação aberta foi o primeiro passo para a análise. Os dados foram analisados linha a linha, sendo cada fala do entrevistado convertida em códigos antes de serem agrupadas por semelhanças e diferenças. Estes agrupamentos geraram conceitos abstratos. O segundo passo da análise foi a codificação axial, na qual os dados continuam submetidos à constante comparação e reorganização. Os conceitos elaborados foram novamente agrupados formando as categorias. A codificação seletiva é a busca e desenvolvimento do fenômeno ou categoria central, sustentada pela interação das demais categorias ${ }^{(12)}$.

A partir disso alcançou-se a saturação dos dados que foram apresentados segundo o Modelo Paradigmático, ou seja, as categorias foram organizadas em Condições Causais, Condições Intervenientes, Contexto, Estratégias de Ação e Interação e Consequências ${ }^{(12)}$.

Do processo analítico, emergiu o fenômeno Vislumbrando as relações e interações do Agente Comunitário na melhoria da assistência à saúde, como uma rede complexa, sustentado pelas categorias: Reconhecendo a comunidade e a Unidade Local de Saúde como espaço para relações e interações, Concebendo a qualidade das relações com a equipe e comunidade, Valorizando seu trabalho como ACS, Percebendo o Enfermeiro como mediador da relação e interação, Sendo o elo entre a comunidade $e$ a equipe, e Favorecendo a assistência através das boas relações.

Reconhecendo a importância e a relevância da primeira categoria, vinculada ao componente "contexto" da TFD, optou-se por apresentá-loa e discutí-la detalhadamente, por exigir um olhar aprofundado sobre o cenário de atuação dos ACS.

Ressalta-se que a pesquisa atendeu os aspectos éticos da resolução 196/96 do Conselho Nacional de Saúde (CEP), sendo o projeto submetido e aprovado no Comitê de Ética em Pesquisa com Seres Humanos da Universidade Federal de Santa Catarina (CEP/UFSC), sob o protocolo número 397/08. Visando garantir o anonimato dos participantes, utilizaram-se pseudônimos associando a letra $\mathrm{P}$ e números arábicos em ordem crescente: P1, P2, P3,..., P21.

\section{RESULTADOS}

A categoria Reconhecendo a comunidade e a Unidade Local de Saúde como espaço para relações e interações é constituía por três subcategorias a seguir apresentadas.

\section{Abordando a comunidade por meio da visita do- miciliar}

Esta subcategoria apresenta a principal ferramenta de atuação do ACS. A visita domiciliar é percebida como elemento chave para criação de vínculo com a comunidade, pois esta permite $\mathrm{o}$ atendimento à localidades e regiões de difícil acesso por outros setores públicos, como também permite o reconhecimento da realidade do ambiente familiar. A visita domiciliar é uma ferramenta da ESF que mantém a comunidade vinculada à Unidade Local de Saúde (ULS) através do cuidado prestado pelo ACS.

De forma mais específica, é pontuado pelos participantes que a visita domiciliar se dá por meio do diálogo e da conversa com a comunidade, visando extrair informações relevantes para a equipe de saúde ou com significativa importância para o usuário. Para alguns ACS, a visita domiciliar vai além de uma atividade laboral formal, pois se sentem como se não estivessem trabalhando, mas em um momento de interação com um amigo.

$\mathrm{Na}$ necessidade de falar sobre algum assunto mais íntimo ou secreto, o usuário e o ACS procuram por um local mais reservado para manter a discrição. Entretanto, mesmo que a relação seja considerada harmoniosa com a maioria dos usuários, de forma geral, os ACS sentem-se mais seguros e confortáveis em atuar na parte externa da casa. Quando conhecem a família, e percebem a necessidade de um período de acompanhamento, permitem-se entrar na casa, como ilustram as falas:

Eu vou e eles já estão me esperando. Aí eu chego lá, brinco, converso, tem aquelas pessoas de idade, e também somos psicólogos porque eles contam aquelas histórias todas e tu ouves. Ai eu pergunto se está tomando o medicamento, aquelas perguntas que é para a gente fazer. (P4)

Eu, geralmente, não gosto de entrar na casa porque para mim é muito intimo, sabe? Eu prefiro ficar mais fora, eu tenho mais segurança. Quando tenho oportunidade ou vejo que é necessário entro para verificar se existe algum problema. (P8) 
Os participantes destacam que a visita domiciliar é uma oportunidade de escuta ativa para identificação das reais necessidades da comunidade, independente do nível socioeconômico das famílias. Para se inserir no contexto do usuário de saúde, utilizam-se brincadeiras e conversas descontraídas, alcançando desta forma a realização de mais visitas domiciliares do que o preconizado. A partir deste processo, o ACS consegue fazer com que o usuário revele alguns problemas da sua família, mesmo que para alcançar as informações exija muito tempo e insistência.

Assim, os ACS confirmam que se sentem à vontade para conversar sobre assuntos mais íntimos com os usuários, pois percebem que conseguem atuar principalmente em situações de mediação de conflitos na família, orientações em saúde e sobre o funcionamento do posto de saúde. Ainda, reforçam que convencem alguns usuários a retomarem seus tratamentos ou a realizarem ações preventivas rotineiras, como vacinas e exames preventivos.

Com a convivência diária com as pessoas da comunidade, os ACS afirmam que se envolvem muito com os problemas dos usuários. Embora estejam sempre disponíveis, quando percebem que não estão bem o suficiente para visitar uma família com dificuldades sérias, preferem adiar a visita domiciliar para outra data, visando ofertar atenção mais qualificada aos usuários.

Durante o trabalho, os ACS se percebem realizando atividades fora de sua atribuição direta, como a entrega de exames e medicações, solicitação e entrega de fraldas, marcação de consultas. Entretanto, estas ações estão inseridas em um contexto especial, uma vez que os usuários envolvidos possuem limitações importantes para o alcance de suas necessidades. Muitos são acamados ou com dificuldade de locomoção, com baixa condição econômica, idosos que moram sozinhos, dentre outros. Essa demanda assumida por alguns ACS é realizada com satisfação por promover o acesso aos serviços do SUS em seus diversos níveis, conforme apresenta o seguinte depoimento:

Gosto de ajudar as pessoas com mais necessidade. Vejo que muitos usuários não têm carro, moram sozinhos, são idosos ou acamados. Então, sempre levo as coisas que eles precisam para fazer o curativo ou as fraldas, seja o que for. Sinto-me bem sabendo que eles não passarão necessidade. (P18)

No que tange ao acesso, os participantes citam a realização de visitas domiciliares no comércio local.
Alguns ACS reforçam que consideram o comércio como uma casa de usuário, realizando visitas frequentes, estabelecendo uma relação próxima e disponibilizando-se para auxiliar em dúvidas. Outros indicam que a visita ao comércio é realizada com menos frequência, com ênfase na divulgação das campanhas do Ministério da Saúde ou epidemias.

\section{Compartilhando informações nas reuniões e grupos}

Os participantes percebem as reuniões como encontros para o planejamento das ações da equipe de saúde e troca de informações sobre a comunidade. Em especial, os ACS consideram que a participação nas reuniões é sua principal atividade quando estão na ULS.

Segundo os participantes, o ACS contribui, significativamente, no esclarecimento de dúvidas sobre as famílias e trazendo dados adicionais. As informações sobre a comunidade são de grande valia para a organização da dinâmica de trabalho e planejamento, no entanto a participação ativa nesse processo é influenciada em parte pela personalidade dos ACS, quando mais comunicativo, maior seu engajamento no planejamento das atividades.

Os entrevistados percebem que a troca de informações entre os profissionais modificam a conduta de todos os membros da equipe, pois aprimoram a abordagem, elegem as prioridades de atenção à saúde e elaboram estratégias para o alcance de suas metas. Em alguns problemas, os ACS percebem que contribuem menos ou não tem o que fazer, pois estão fora de sua área de atuação, então reportam a situação aos enfermeiros e médicos.

Percebem que as reuniões são, na maioria das vezes, descontraídas, com potencial formativo, pois ampliam o conhecimento sobre diversos assuntos e fluxos do setor saúde. Os ACS sentem-se respeitados e valorizados pelas informações que compartilham e sugestões de atuação prática. Entretanto, a equipe percebe que a participação deve ser incentivada, pois geralmente os ACS respondem a estímulos, sem ter iniciativa ou participação espontânea na reunião, como descreve o depoimento a seguir:

Todo esforço do grupo em tornar nossa prática mais dialogada contribui para o relacionamento com a comunidade. Mas, ainda percebo os agentes comunitários em um papel mais passivo. Para mudar essa postura, sempre solicitamos sua opinião e questionamos sobre a melhor abordagem para a família ou usuário. (P15) 
A relação dos ACS com a comunidade na ULS se dá principalmente nos grupos terapêuticos. Outros locais de encontro são as escolas e creches da comunidade, onde são desenvolvidas atividades de promoção e prevenção em saúde bucal. Nesses encontros, realizam atividades teatrais e auxiliam em supervisão direta, como na higiene dental de crianças nas escolas, por exemplo.

\section{Percebendo as festinhas como momentos de inte- ração no posto}

Os ACS destacam que os momentos de interação informal, como festinhas e lanches coletivos, são importantes momentos de formação de vínculo entre os profissionais, por tornar o grupo mais unido e harmonioso.

Eles ressaltam que a relação com o enfermeiro é mais próxima, quando comparada com os demais profissionais, por ser um supervisor direto de suas ações. Nesse sentido, perceberam que outras formas de aproximação seriam necessárias para aproximar os diferentes profissionais que trabalham na ULS. Os ACS relacionam o distanciamento com os outros membros da equipe à sua atuação laboral, pois passam mais tempo na comunidade junto aos usuários, como se observa no depoimento a seguir:

Acredito que realizar festinhas para comemorar conquistas coletivas e aniversários dos colegas de trabalho é um importante canal para nos colocar em contato. Fico feliz quando olho para trás e lembro que mal tinha contato com o médico ou com o dentista porque tinha vergonha de falar com eles, e hoje somos mais próximos para tirar qualquer dúvida. (P7)

Os encontros descontraídos compreendem comemoração de aniversários, comemoração das metas alcançadas ou finalização de grandes trabalhos, como as campanhas. Ainda, é relatado que essas relações ampliam o contato entre os profissionais, permitindo maior contato com os supervisores e coordenador, potencializando a integração da equipe na realização das atividades coletivas.

\section{DISCUSSÃO}

A visita domiciliar é considerada como uma tecnologia de interação no cuidado, sendo uma ferramenta de intervenção fundamental na prática dos profissionais da equipe da ULS, permitindo com que o visitador conheça a realidade que o cliente está inserido. A visita domiciliar é uma atribuição comum a todos os profissionais da ESF. No entanto, é o ACS que realiza as visitas domiciliares com maior frequência, constituindo-se como seu principal instrumento de trabalho ${ }^{(13)}$.

Além da visita domiciliar, os ACS fazem o mapeamento das áreas; cadastramento das famílias no Sistema de Informação da Atenção Básica; identificação das microáreas de risco; e estímulo às ações coletivas. $\mathrm{Ou}$ seja, ele promove reuniões com grupos diferenciados como gestantes, mães, idosos, além de atuar como elo entre a comunidade e o serviço de saúde, estimulando-a à participação social ${ }^{(14)}$. O trabalho do ACS também está baseado em ações educativas e preventivas que buscam fortalecer a organização, a valorização e o desenvolvimento comunitário bem como o vínculo do sistema de saúde com a comunidade ${ }^{(15)}$.

O ACS é um profissional da comunidade e a representa dentro do serviço de saúde, fazendo a aproximação entre o saber técnico da equipe de saúde ao saber popular presente nos diversos grupos sociais da comunidade $^{(16)}$. Em pesquisa desenvolvida no Ceará, os ACS relataram estarem satisfeito em poder realizar atividades voltadas à caridade e a possibilidade de conhecer as pessoas na comunidade onde moram ${ }^{(17)}$. Eles identificam-se tanto com o trabalho realizado que não se sentem trabalhando ao fazer as visitas domiciliares. No momento da visita domiciliar, o agente tem a oportunidade de compreender o modo de vida do usuário; conhecer o ambiente familiar e as relações intrafamiliares; abordar questões que vão além da doença física e contemplem também problemas sociais e emocionais; proporcionando, dessa forma, orientações voltadas para as reais necessidades de saúde do usuário, buscando singularidades na forma de cuidar ${ }^{(13)}$.

Na ULS estudada, as reuniões e os grupos foram considerados pelos participantes como espaços de compartilhamentos de informações. É importante destacar que as reuniões são momentos de interação, em que os profissionais se encontram para trocar informações sobre a comunidade, expressarem os problemas cotidianos e o andamento do serviço, bem como um momento de construção das relações interpessoais com os diversos profissionais que atuam na $\operatorname{ULS}^{(18)}$.

Desse modo, ressalta-se que é fundamental que as reuniões proporcionem a interação entre os membros da equipe de saúde, de modo que possam conhecer melhor as atividades diárias de cada trabalhador, tornando-se um espaço para compartilhar seus anseios, dúvidas e expectativas. $E$, ainda, permita a discussão de 
problemas identificados na prática cotidiana da equipe com a construção de consensos para sua resolução, mesmo que seja em caráter temporário ${ }^{(10)}$.

A perspectiva multidisciplinar possibilita um trabalho mais articulado da equipe de saúde, no que diz respeito à compreensão dos profissionais sobre o trabalho de seus colegas, como no que diz respeito à qualidade do trabalho final, desenvolvido em conjunto. Esta prática coloca-se como potencializadora na conexão entre os diversos olhares profissionais, possibilitando outras formas de relação entre os trabalhadores envolvidos no cuidado ${ }^{(9)}$.

O trabalho multidisciplinar ajuda a aprofundar a relação da equipe, as reuniões formais e informais tornam-se momentos ricos pela troca de informações e vivências dos diferentes profissionais existentes na ESF. O fato de estarem todos juntos intensifica esta interação, auxiliando e melhorando o planejamento das ações desenvolvidas na ULS frente às necessidades encontradas na população. A contribuição de cada membro da equipe fortalece as ações planejadas por conter diversos olhares e saberes profissionais. A importância da interação das pessoas nas organizações e nas equipes deriva do fato de que grande parte do sucesso alcançado pelas equipes pode ser explicado pelo grau de eficácia na administração das relações entre cargos, indivíduos e seu impacto no desempenho do grupo ${ }^{(19)}$.

\section{CONSIDERAÇÕES FINAIS}

Conclui-se que o contexto no qual se desenvolve a rede de relações e interações dos ACS em uma ESF torna-se amplo, pois suas atividades são desenvolvidas diretamente ligadas à comunidade, extrapolando os limites da ULS. Abrangem a comunidade, por meio das visitas domiciliares, bem como o contexto institucional, em encontros formais e informais, que favorecem a relação com a equipe multiprofissional, repercutindo positivamente nas atividades diárias da unidade.

Destaca-se que o trabalho do ACS, por residir na área de atuação e conhecer bem a sua realidade, torna-se um forte elo de ligação entre a ULS e a comunidade. Esta interação acontece, na maioria das vezes, através da visita domiciliar, momento em que o agente consegue coletar o maior número de informações necessárias para subsidiar o trabalho da equipe de saúde, em especial do enfermeiro, com o qual o ACS já estabelece importante via de comunicação.

Os dados coletados pelos ACS devem ser compartilhados entre todos os profissionais da ESF para que possam trazer a realidade da comunidade para a
ULS. Nesse sentido, o enfermeiro deve incentivar a participação e protagonismo do ACS respeitando suas características pessoais. As reuniões e os grupos são momentos ideais para ocorrer a interação entre a equipe de saúde por ser um momento onde os profissionais se reúnem e compartilham informações necessárias para a melhoria da saúde da população atendida. Em alguns casos, ocorre o distanciamento do ACS dos outros profissionais por sua atuação laboral desenvolver-se mais na comunidade, porém os encontros informais promovidos pela equipe proporcionam momentos de interação e formação de vínculo, como também aproximam os ACS da equipe.

É fundamental reconhecer e valorizar a importância das atividades desenvolvidas pelos ACS, como a visita domiciliar para a continuidade positiva e exitosa da ESF.

Desse modo, espera-se que os achados desta pesquisa contribuam para futuras reflexões, subsidiem questionamentos a respeito das estratégias de interação nos contextos de atuação dos ACS e a equipe de saúde, como também proponham mudanças e coloquem em prática ações reconhecedoras do trabalho desses profissionais.

\section{REFERÊNCIA}

1. Mattioni FC, Budó MLD, Schimith MD. Exercising integrality in a family health strategy team: knowledge and practices. Texto Contexto Enferm. [Internet] 2011 [acesso em: $10 \mathrm{fev} 2013$ ]; 20(2). Disponível em: http://www.scielo.br/pdf/tce/v20n2/a07v20n2. pdf. Epub Jun, 2011. http://dx.doi.org/10.1590/S010407072011000200007

2. Uchimura KY, Bosi MLM. Skills and competencies among workers in the Family Health Strategy. Interface Comun Saude Educ. [Internet] 2012 [acesso em: $10 \mathrm{fev}$ 2013]; 16(40). Disponível: http://www.scielo.br/pdf/ icse/v16n40/en_aop0812.pdf. Epub Apr 03, 2012. http:// dx.doi.org/10.1590/S1414-32832012005000006

3. Senna MCM, Costa AM, Silva LN. Health care in big cities: challenges to the consolidation of SUS. Sociedade debate [Internet] 2012 [acesso em: 12 fev 2013];16(1). Disponível em: http:/www.rle.ucpel.tche.br/index.php/ rsd/article/view/341/299

4. Kawata LS, Mishima SM, Chirelli MQ, Pereira MJB. Nursing daily work in family health: using management tools. Texto Contexto Enferm. [Internet] 2009 [acesso em: 12 fev 2013];18(2). Disponível em: http://www. scielo.br/pdf/tce/v18n2/15.pdf. Epub Jun, 2009. http:// dx.doi.org/10.1590/S0104-07072009000200015 
5. Lanzoni GMM, Meirelles BHS. A network of relationships and interactions of the health care team in primary care, and implications for nursing. Acta Paul Enferm. [Internet] 2012 [acesso em: 12 fev 2013]; 25(3). Disponível em: http://www.scielo.br/pdf/ape/ v25n3/en_v25n3a23.pdf. Epub Mar, 2012. http://dx.doi. org/10.1590/S0103-21002012000300023

6. Lino MM, Lanzoni GMM, Schveitzer MC, Albuquerque GL. Permanent Education to Communitarian Agents of Health: needs and challenges. Saúde Transform Soc. [Internet] 2011 [acesso em: 12 fev 2013]; 1(3). Disponível em: http://periodicos.incubadora.ufsc.br/index.php/ saudeetransformacao/article/view/653/877

7. Costa EM, Ferreira, DLA. Community health agents perceptions and motivations about the labor process in Teresina, Piauí. Trab. Educ. Saúde. [Internet] 2012 [acesso em: 12 fev 2013];9(3). Disponível em: www. scielo.br/pdf/tes/v9n3/v9n3a07.pdf. Epub Nov, 2012. http://dx.doi.org/10.1590/S1981-77462011000300007

8. Santana JCB, Vasconcelos AL, Martins CV, Barros JV, Soares JM, Dutra BS. Community health agent: family health strategy perceptions. Cogitare enferm. 2009;14(4):645-52.

9. Matos E, Pires DEP, Sousa GW. Work relationships among interdisciplinary teams: contributions for new methods of organization in health work. Rev Bras Enferm [Internet]. 2010 [acesso em: 12 fev 2013];63(5). Disponível em: http://www.scielo.br/pdf/reben/ v62n6/a10v62n6.pdf. Epub Dec, 2010. http://dx.doi. org/10.1590/S0034-71672009000600010

10. Colomé ICS, Lima MADS, Davis R. Nurses' view on the coordination of health actions among professionals of family health teams. Rev Esc Enferm USP. [Internet] 2008 [acesso em: 12 fev 2013] 42(2).Disponível em: http://www.scielo.br/pdf/reeusp/v42n2/a06.pdf. Epub Jun, 2008. http://dx.doi.org/10.1590/S008062342008000200007

11. Barbosa SP, Aguiar AC. Factors influencing the drop-out rate of nurses in the family health strategy in Ipatinga, Minas Gerais, Brazil. Rev APS. [Internet] 2008 [acesso em 12 fev 2013];11(4). Disponível em: http://www.aps. ufjf.br/index.php/aps/article/view/155/135

12. Baggio MA, Erdmann AL. Theory based on the data or Grounded Theory and its use in nursing research in Brazil. Referencia. [Internet] 2011 [acesso em: $12 \mathrm{fev}$ 2013];3(3). Disponível em: http://www.esenfc.pt/site/ index.php? module $=$ rr\&target $=$ publicationDetails\&pe squisa $=\&$ id_artigo $=2227 \&$ id_revista $=9 \&$ id_edicao $=35$
13. Kebian LVA, Acioli S. Visita domiciliar: espaço de práticas de cuidado do enfermeiro e do agente comunitário de saúde. Rev. enferm. UERJ. [Internet] 2011 [acesso em: 26 abr 2013];19(3). Disponível em: http://www.facenf.uerj.br/v19n3/v19n3a11.pdf

14. Ministério da Saúde (BR). Portaria no 2.488, de 21 de outubro de 2011: aprova a Política Nacional de Atenção Básica, estabelecendo a revisão de diretrizes e normas para a organização da Atenção Básica, para a Estratégia Saúde da Família (ESF) e o Programa de Agentes Comunitários de Saúde (PACS). Diário Oficial da União, [Internet] 24 out 2011. [Acesso em: 12 fev 2013] Disponível: http://bvsms.saude.gov.br/bvs/saudelegis/ gm/2011/prt2488_21_10_2011.html

15. Galavote HS, Prado TN, Maciel ELN, Lima RCD. Disclosing the work processes of the community health agents on the Family Health Strategy in Vitória (ES, Brazil). Cien Saude Coletiva [Internet] 2011 [acesso em: 12 fev 2013];16(1).Disponível em: http://www.scielo.br/ pdf/csc/v16n1/v16n1a26.pdf. Epub Nov, 2011. http:// dx.doi.org/10.1590/S1413-81232011000100026

16. Muller B, Barradas D, Costa MAR, Cambiriba MS. The professionalization of the community healthcare assistant from the perspective of health promotion. Cogitare enferm. 2012;17(1):171-4.

17. Oliveira DT, Ferreira PJO, Mendonça LBA, Oliveira HS. Community health assistants' perceptions about their role in the family health strategy. Cogitare enferm. 2012;17(1):132-7.

18. Peres CRFB, Caldas Júnior AL, Silva RF, Marin MJS. O Agente Comunitário de Saúde frente ao processo de trabalho em equipe: facilidades e dificuldades. Rev Esc Enferm USP [Internet]. 2011 [acesso em: 29 abr 2013] 45(4). Disponível em: http://www.scielo.br/pdf/reeusp/ v45n4/v45n4a16.pdf.

19. Matos ACA, Farias LGQ. Diagnosis of interaction in the family health teamwork in Ibiçaraí, Bahia. Gestão Contemporânea. [Internet] 2009 [acesso em: 12 fev 2013]; (6)6. Disponível em: http://seer2.fapa.com.br/ index.php/arquivo/article/view/12/8. 